\title{
A Note on Stability of a Linear Functional Equation of Second Order Connected with the Fibonacci Numbers and Lucas Sequences
}

\author{
Janusz Brzdęk ${ }^{1}$ and Soon-Mo Jung ${ }^{2}$ \\ ${ }^{1}$ Department of Mathematics, Pedagogical University, Podchorażych 2, 30-084 Kraków, Poland \\ ${ }^{2}$ Mathematics Section, College of Science and Technology, Hongik University, \\ 339-701 Jochiwon, Republic of Korea
}

Correspondence should be addressed to Soon-Mo Jung, smjung@hongik.ac.kr

Received 26 April 2010; Accepted 15 July 2010

Academic Editor: Ram N. Mohapatra

Copyright (c) 2010 J. Brzdęk and S.-M. Jung. This is an open access article distributed under the Creative Commons Attribution License, which permits unrestricted use, distribution, and reproduction in any medium, provided the original work is properly cited.

\begin{abstract}
We prove the Hyers-Ulam stability of a second-order linear functional equation in single variable (with constant coefficients) that is connected with the Fibonacci numbers and Lucas sequences. In this way we complement, extend, and/or improve some recently published results on stability of that equation.
\end{abstract}

\section{Introduction}

In this paper $\mathbb{C}, \mathbb{R}, \mathbb{Z}$, and $\mathbb{N}$ stand, as usual, for the sets of complex numbers, real numbers, integers, and positive integers, respectively. Let $S$ be a nonempty set, $\xi: S \rightarrow S, X$ be a Banach space over a field $\mathbb{K} \in\{\mathbb{C}, \mathbb{R}\}, p, q \in \mathbb{K}, q \neq 0$, and $a_{1}, a_{2}$ denote the complex roots of the equation

$$
x^{2}-p x+q=0
$$

Moreover, $\xi^{0}(x)=x, \xi^{n+1}(x)=\xi\left(\xi^{n}(x)\right)$, and (only for bijective $\left.\xi\right) \xi^{-n-1}(x)=\xi^{-1}\left(\xi^{-n}(x)\right)$ for $x \in S$ and $n \in \mathbb{N}_{0}:=\mathbb{N} \cup\{0\}$.

The problem of stability of functional equations was motivated by a question of Ulam asked in 1940 and a solution to it by Hyers published in [1]. Since then numerous papers have been published on that subject and we refer to [2-7] for more details, some discussions 
and further references; for examples of very recent results see, for example, [8-12]. Jung has proved in [5] (see also [13]) some results on solutions and stability of the functional equation

$$
f(x)=p f(\xi(x))-q f\left(\xi^{2}(x)\right)
$$

in the case where $S=\mathbb{R}$ and $\xi(x)=x-1$ for $x \in \mathbb{R}$. The result on stability (see [5, Theorem 3.1]) can be stated as follows.

Theorem 1.1. Let $p, q \in \mathbb{R}, p^{2}-4 q \neq 0,0<\left|a_{2}\right|<1<\left|a_{1}\right|, a_{1}, a_{2} \in \mathbb{K}, \varepsilon>0$, and $g: \mathbb{R} \rightarrow X$ satisfy the inequality

$$
\sup _{x \in \mathbb{R}}\|g(x)-p g(x-1)+q g(x-2)\| \leq \varepsilon
$$

Then there is a unique solution $f: \mathbb{R} \rightarrow X$ of the functional equation

$$
f(x)=p f(x-1)-q f(x-2)
$$

with

$$
\sup _{x \in \mathbb{R}}\|g(x)-f(x)\| \leq \frac{\left(\left|a_{1}\right|-\left|a_{2}\right|\right) \varepsilon}{\left|a_{1}-a_{2}\right|\left(\left|a_{1}\right|-1\right)\left(1-\left|a_{2}\right|\right)} .
$$

If $S=\mathbb{N}_{0}$ and $p, q \in \mathbb{Z}$, then solutions $x: \mathbb{N}_{0} \rightarrow \mathbb{Z}$ of the difference equation (1.4) are called the Lucas sequences (see, e.g., [14]); in some special cases they are called with specific names; for example; the Fibonacci numbers $(p=1, q=-1, x(0)=0$ and $x(1)=1)$, the Lucas numbers $(p=1, q=-1, x(0)=2$ and $x(1)=1)$, the Pell numbers $(p=2, q=-1, x(0)=0$ and $x(1)=1)$, the Pell-Lucas (or companion Lucas) numbers $(p=2, q=-1, x(0)=2$ and $x(1)=2)$, and the Jacobsthal numbers $(p=1, q=-2, x(0)=0$ and $x(1)=1)$.

For some information and further references concerning the functional equations in single variable we refer to [15-17]; for an ample survey on stability results for those equations see [2]. Let us mention yet that the problem of stability of functional equations is connected to the notions of controlled chaos (see [18]) and shadowing (see [19-21]).

Remark 1.2. If $\xi$ is bijective, then, with $\eta:=\xi^{-1},(1.2)$ can be written in the following equivalent form:

$$
f\left(\eta^{2}(x)\right)=p f(\eta(x))-q f(x)
$$

Clearly, (1.1) is the characteristic equation of (1.6).

In view of Remark 1.2, from [22, Theorem 2] (see also [23]) the following stability result, concerning (1.2), can be derived. 
Theorem 1.3. Let $\left|a_{i}\right| \neq 1$ for $i=1,2$, $\xi$ be bijective, $\varepsilon>0$, and $g: S \rightarrow X$ satisfy the inequality

$$
\sup _{x \in S}\left\|g(x)-p g(\xi(x))+q g\left(\xi^{2}(x)\right)\right\| \leq \varepsilon
$$

Then there is a unique solution $f: S \rightarrow X$ of (1.2) with

$$
\sup _{x \in S}\|g(x)-f(x)\| \leq \frac{\varepsilon}{\left|\left(\left|a_{1}\right|-1\right)\left(\left|a_{2}\right|-1\right)\right|}
$$

Theorem 1.3 appears to be much more general than Theorem 1.1 (obtained by a different method of proof). But on the other hand, estimation (1.5) is significantly sharper than (1.8) in numerous cases (take, e.g., $a_{1}=1+1 / n$ and $a_{2}=-1+1 / n$, with some large $n \in \mathbb{N}$ ). Therefore, there arises a natural question if the method applied in [5] can be modified so as to prove a more general equivalent of Theorem 1.3, but with an estimation better than (1.8). In this paper, we show that this is the case. Namely, we prove the following.

Theorem 1.4. Let $\varepsilon>0$ and $g: S \rightarrow X$ satisfy inequality (1.7). Suppose that $a_{1} \neq a_{2}$ and one of the following two conditions is valid:

(a) $\left|a_{i}\right|<1$ for $i=1,2$;

( $\beta)\left|a_{i}\right| \neq 1$ for $i=1,2$ and $\xi$ is bijective.

Then there exists a solution $F: S \rightarrow X$ of (1.2) such that

$$
\sup _{x \in S}\|g(x)-F(x)\| \leq \frac{\varepsilon}{\left|a_{1}-a_{2}\right|}\left(\frac{\left|a_{1}\right|}{|| a_{1}|-1|}+\frac{\left|a_{2}\right|}{|| a_{2}|-1|}\right) .
$$

Moreover, if condition $(\beta)$ is valid, then there exists exactly one solution $f: S \rightarrow X$ of (1.2) with $\sup _{x \in S}\|g(x)-f(x)\|<\infty$.

Remark 1.5. Note that, for bijective $\xi$, Theorem 1.4 improves estimation (1.8) in some cases (take, e.g., $a_{1}=3 / 2, a_{2}=-3 / 2$, or $a_{1}=1 / 2, a_{2}=-1 / 2$ ); however, in some other situations (e.g., $a_{1}=3, a_{2}=-3$ ), estimation (1.8) is better. Theorem 1.4 also complements Theorem 1.3 because $\xi$ can be quite arbitrary in the case of $(\alpha)$.

\section{Proof of Theorem 1.4}

Clearly, $a_{1}+a_{2}=p$ and $a_{1} a_{2}=q$. We start with the case $\mathbb{K}=\mathbb{C}$.

Fix $i \in\{1,2\}$ and first assume that $\left|a_{i}\right|<1$. Write

$$
A_{k}^{i}(x):=a_{i}^{k}\left[g\left(\xi^{k}(x)\right)-\left(p-a_{i}\right) g\left(\xi^{k+1}(x)\right)\right], \quad x \in S, k \in \mathbb{N}_{0}
$$


Then, for each $k \in \mathbb{N}_{0}$ and $x \in S$,

$$
\begin{aligned}
A_{k}^{i}(x)-A_{k+1}^{i}(x)= & a_{i}^{k}\left[g\left(\xi^{k}(x)\right)-\left(p-a_{i}\right) g\left(\xi^{k+1}(x)\right)\right] \\
& -a_{i}^{k+1}\left[g\left(\xi^{k+1}(x)\right)-\left(p-a_{i}\right) g\left(\xi^{k+2}(x)\right)\right] \\
= & a_{i}^{k}\left[g\left(\xi^{k}(x)\right)-p g\left(\xi^{k+1}(x)\right)+q g\left(\xi^{k+2}(x)\right)\right],
\end{aligned}
$$

whence

$$
\left\|A_{k}^{i}(x)-A_{k+1}^{i}(x)\right\| \leq\left|a_{i}\right|^{k} \varepsilon
$$

and consequently

$$
\left\|A_{k}^{i}(x)-A_{k+n}^{i}(x)\right\| \leq \sum_{j=k}^{k+n-1}\left|a_{i}\right|^{j} \varepsilon, \quad n \in \mathbb{N}_{0} .
$$

This means that, for each $x \in S,\left\{A_{n}^{i}(x)\right\}_{n \in \mathbb{N}}$ is a Cauchy sequence and therefore there exists the limit $F_{i}(x)=\lim _{n \rightarrow \infty} A_{n}^{i}(x)$. Further, for every $x \in S$,

$$
\begin{aligned}
p F_{i}(\xi(x))-q F_{i}\left(\xi^{2}(x)\right) & =p a_{i}^{-1} \lim _{n \rightarrow \infty} A_{n+1}^{i}(x)-q a_{i}^{-2} \lim _{n \rightarrow \infty} A_{n+2}^{i}(x) \\
& =p a_{i}^{-1} F_{i}(x)-q a_{i}^{-2} F_{i}(x) \\
& =F_{i}(x)
\end{aligned}
$$

and, by (2.4) with $k=0$ and $n \rightarrow \infty$,

$$
\sup _{x \in S}\left\|g(x)-\left(p-a_{i}\right) g(\xi(x))-F_{i}(x)\right\| \leq \frac{\varepsilon}{|1-| a_{1}||}
$$

Now, assume that $\left|a_{i}\right|>1$. This means that $\xi$ is bijective. Let

$$
A_{k}^{i}(x):=a_{i}^{-k}\left[g\left(\xi^{-k}(x)\right)-\left(p-a_{i}\right) g\left(\xi^{-k+1}(x)\right)\right], \quad x \in S, k \in \mathbb{N}_{0}
$$

Then, for each $k \in \mathbb{N}$ and $x \in S$,

$$
\begin{aligned}
A_{k}^{i}(x)-A_{k-1}^{i}(x)= & a_{i}^{-k}\left[g\left(\xi^{-k}(x)\right)-\left(p-a_{i}\right) g\left(\xi^{-k+1}(x)\right)\right] \\
& -a_{i}^{-k+1}\left[g\left(\xi^{-k+1}(x)\right)-\left(p-a_{i}\right) g\left(\xi^{-k+2}(x)\right)\right] \\
= & a_{i}^{-k}\left[g\left(\xi^{-k}(x)\right)-p g\left(\xi^{-k+1}(x)\right)+q g\left(\xi^{-k+2}(x)\right)\right]
\end{aligned}
$$


and next, by (1.7),

$$
\left\|A_{k}^{i}(x)-A_{k-1}^{i}(x)\right\| \leq\left|a_{i}\right|^{-k} \varepsilon
$$

Hence,

$$
\left\|A_{k}^{i}(x)-A_{k+n}^{i}(x)\right\| \leq \sum_{j=1}^{n}\left|a_{i}\right|^{-j-k} \varepsilon, \quad n \in \mathbb{N}_{0}, x \in S .
$$

So, for each $x \in S,\left\{A_{n}^{i}(x)\right\}_{n \in \mathbb{N}}$ is a Cauchy sequence and consequently there exists the limit $F_{i}(x)=\lim _{n \rightarrow \infty} A_{n}^{i}(x)$. Note that, for every $x \in S$, (2.5) holds and, by (2.10) with $k=0$ and $n \rightarrow \infty$,

$$
\sup _{x \in S}\left\|g(x)-\left(p-a_{i}\right) g(\xi(x))-F_{i}(x)\right\| \leq \frac{\varepsilon\left|a_{i}\right|^{-1}}{1-\left|a_{i}\right|^{-1}}=\frac{\varepsilon}{\left|a_{i}\right|-1}
$$

Thus, we have proved that, for $i=1,2$, inequality (2.6) holds and $F_{i}$ is a solution to (1.2). Define $F: S \rightarrow X$ by

$$
F(x):=\frac{a_{1}}{a_{1}-a_{2}} F_{1}(x)-\frac{a_{2}}{a_{1}-a_{2}} F_{2}(x), \quad x \in S .
$$

Then, for $x \in S$, it follows from (2.5) that

$$
\begin{aligned}
p F(\xi(x))-q F\left(\xi^{2}(x)\right)= & \frac{a_{1}}{a_{1}-a_{2}}\left[p F_{1}(\xi(x))-q F_{1}\left(\xi^{2}(x)\right)\right] \\
& -\frac{a_{2}}{a_{1}-a_{2}}\left[p F_{2}(\xi(x))-q F_{2}\left(\xi^{2}(x)\right)\right] \\
= & F(x)
\end{aligned}
$$

and, by (1.1) and (2.6),

$$
\begin{aligned}
\|g(x)-F(x)\| & =\frac{1}{\left|a_{1}-a_{2}\right|}\left\|\left(a_{1}-a_{2}\right) g(x)-a_{1} F_{1}(x)+a_{2} F_{2}(x)\right\| \\
& \leq \frac{\varepsilon}{\left|a_{1}-a_{2}\right|}\left(\frac{\left|a_{1}\right|}{|| a_{1}|-1|}+\frac{\left|a_{2}\right|}{|| a_{2}|-1|}\right) .
\end{aligned}
$$

In the case where $\xi$ is bijective, the uniqueness of $F$ results from [22, Proposition 1], in view of Remark 1.2. 
Now, assume that $\mathbb{K}=\mathbb{R}$. Then (see, e.g., [24, page 39], [25], or [26, 27, 1.9.6, page 66]) $\mathrm{X}^{2}$ is a complex Banach space with the linear structure and the Taylor norm $\|\cdot\|_{T}$ given by

$$
\begin{gathered}
(x, y)+(z, w):=(x+z, y+w) \quad \text { for } x, y, z, w \in X, \\
(\alpha+i \beta)(x, y):=(\alpha x-\beta y, \beta x+\alpha y) \quad \text { for } x, y \in X, \alpha, \beta \in \mathbb{R}, \\
\|(x, y)\|_{T}:=\sup _{0 \leq \theta \leq 2 \pi}\|(\cos \theta) x+(\sin \theta) y\| \quad \text { for } x, y \in X .
\end{gathered}
$$

Clearly, $\max \{\|x\|,\|y\|\} \leq\|(x, y)\|_{T} \leq\|x\|+\|y\|$ for all $x, y \in X$.

Define $x: S \rightarrow X^{2}$ by $\chi(x):=(g(x), 0)$ for $x \in S$. Then,

$$
\left\|\chi(x)-p_{X}(\xi(x))+q \chi\left(\xi^{2}(x)\right)\right\|_{T} \leq \varepsilon, \quad x \in S .
$$

So, by the previous part of the proof, there exists a solution $H: S \rightarrow X^{2}$ of (1.2) such that

$$
\|x(x)-H(x)\|_{T} \leq \frac{\varepsilon}{\left|a_{1}-a_{2}\right|}\left(\frac{\left|a_{1}\right|}{|| a_{1}|-1|}+\frac{\left|a_{2}\right|}{|| a_{2}|-1|}\right), \quad x \in S .
$$

Write $p_{i}\left(x_{1}, x_{2}\right):=x_{i}$ for $x_{1}, x_{2} \in X, i=1,2$. Clearly, $F: S \rightarrow X$, given by $F(x):=p_{1}(H(x))$ for $x \in S$, is a solution of (1.2), and (1.9) holds.

It remains to prove the statement concerning uniqueness of $F$. So, let $F_{0}: S \rightarrow X$ be a solution of (1.2) with $\sup _{x \in S}\left\|g(x)-F_{0}(x)\right\|<\infty$. Let $H_{0}(x):=\left(F_{0}(x), p_{2}(H(x))\right)$ for $x \in S$. It is easily seen that $H_{0}$ is a solution of (1.2). Moreover, for every $x \in S$,

$$
\left\|H(x)-H_{0}(x)\right\|_{T}=\left\|F(x)-F_{0}(x)\right\| \leq\|F(x)-g(x)\|+\left\|g(x)-F_{0}(x)\right\| .
$$

Hence, by [22, Proposition 1], $H=H_{0}$, which yields $F_{0}=F$.

\section{Consequences of Theorem 1.4}

Now we present some consequences of Theorem 1.4 and some results from [22, 28, 29].

Theorem 3.1. Let $\varepsilon>0$ and $g: S \rightarrow X$ satisfy (1.7). Suppose that one of the following three conditions is valid:

(i) $\left|a_{i}\right|<1$ for $i=1,2$ and $a_{1} \neq a_{2}$;

(ii) $\left|a_{i}\right| \neq 1$ for $i=1,2$ and $\xi$ is bijective;

(iii) (ii) holds and $a_{1} \neq a_{2}$.

Then there exists a solution $F: S \rightarrow X$ of (1.2) such that

$$
\sup _{x \in S}\|g(x)-F(x)\| \leq M \varepsilon
$$


where

$$
\begin{gathered}
M= \begin{cases}\min \left\{M_{1}, M_{2}\right\}, & \text { if }(\text { i) or (iii) holds, } \\
M_{2}, & \text { if (ii) holds, }\end{cases} \\
M_{1}:=\frac{1}{\left|a_{1}-a_{2}\right|}\left(\frac{\left|a_{1}\right|}{|| a_{1}|-1|}+\frac{\left|a_{2}\right|}{|| a_{2}|-1|}\right), \\
M_{2}:=\frac{1}{\left|\left(\left|a_{1}\right|-1\right)\left(\left|a_{2}\right|-1\right)\right|}
\end{gathered}
$$

Moreover, if $\left|a_{i}\right|<1$ for $i=1,2$, then there exists exactly one solution $f: S \rightarrow X$ of (1.2) such that $\sup _{x \in S}\|g(x)-f(x)\|<\infty$.

Proof. If (i) is valid, then Theorem 1.4 yields (3.1) with $M=M_{1}$. Further, by (1.7),

$$
\sup _{x \in S}\left\|g\left(\xi^{2}(x)\right)-\frac{p}{q} g(\xi(x))+\frac{1}{q} g(x)\right\| \leq \frac{\varepsilon}{|q|}
$$

and $b_{i}:=a_{i} / q=1 / a_{i}$ for $i=1,2$ are roots of the equation

$$
x^{2}-\frac{p}{q} x+\frac{1}{q}=0
$$

Hence, by [22, Theorem 2], there is a solution $F: S \rightarrow X$ of the functional equation

$$
F\left(\xi^{2}(x)\right)=\frac{p}{q} F(\xi(x))-\frac{1}{q} F(x)
$$

such that

$$
\sup _{x \in S}\|g(x)-F(x)\| \leq \frac{\varepsilon /|q|}{\left(\left|a_{1} / q\right|-1\right)\left(\left|a_{2} / q\right|-1\right)}=M_{2} \varepsilon
$$

(The last equality is due to the fact that $q=a_{1} a_{2}$.) It is easily seen that $F$ is a solution to (1.2).

Next, consider the case of (ii). Then, in view of Theorem 1.4, there is a solution $F: S \rightarrow$ $X$ of (1.2) satisfying (3.1) with $M=M_{1}$. Further,

$$
\sup _{x \in S}\left\|g\left(\eta^{2}(x)\right)-p g(\eta(x))+q g(x)\right\| \leq \varepsilon
$$

with $\eta:=\xi^{-1}$. Hence, according to [22, Theorem 2], there exists a function $F: S \rightarrow X$ satisfying (1.6) and inequality (3.1), with $M=M_{2}$. Now, it is enough to note that $F$ is a solution to (1.2), as well.

Finally, if (iii) holds, then it is enough to use [22, Theorem 2] and Theorem 1.4 (the case of $(\beta))$. 
The statement concerning uniqueness results from [22, Proposition 1].

Remark 3.2. If $\left|a_{i}\right|=1$ for some $i \in\{1,2\}$ (or, equivalently, $\left|a_{i}\right|=|q|$ for some $i \in\{1,2\}$ ), then (1.2) can be nonstable, by which we mean that there is a function $g: S \rightarrow X$ such that (1.7) holds with some real $\varepsilon$ and $\sup _{x \in S}\|g(x)-f(x)\|=\infty$ for each solution $f: S \rightarrow X$ of (1.2) (see, e.g., [28], [22, Example 1], or [29]).

Remark 3.3. Note that, in the case where $p, q, a_{1}, a_{2}$ are real numbers, we have

$$
\left(\left|a_{1}\right|-1\right)\left(\left|a_{2}\right|-1\right)=|q|+1-p_{0}
$$

with

$$
p_{0}:=\left|a_{1}\right|+\left|a_{2}\right|= \begin{cases}|p|, & \text { if } q>0, \\ \sqrt{p^{2}-4 q}, & \text { if } q<0 .\end{cases}
$$

\section{Some Critique and Final Remarks}

Functional equation (1.2) has been patterned on difference equation (1.4). However, if we want to apply the results of Theorems 1.1-3.1 to the Lucas sequences we come across two obstacles. The first one concerns the domain of $\xi$ and arises from the difference equation (1.4) being written in "wrong" historical form, inconsistent with the general concept of functional equations. Actually it should be written as the functional equation

$$
f(x+2)=p f(x+1)-q f(x)
$$

which corresponds to (1.6). The second obstacle is connected with the restrictions on $a_{i}$. For some interesting cases (Fibonacci, Lucas, or Pell numbers), we have $\left|a_{1}\right|<1<\left|a_{2}\right|$ (or, if somebody prefers, $\left|a_{2}\right|<1<\left|a_{1}\right|$ ) and such case is not covered if $\xi$ is not bijective (which is the case when $S=\mathbb{N}_{0}$ and $\xi(x)=x-1$ or, equivalently, $\left.\eta(x)=x+1\right)$. All these obstacles can be overcome if, instead of Theorems 1.1-3.1, we use the following result derived from [29, Theorem 2.3].

Proposition 4.1. Let $\varepsilon>0, a_{i} \in \mathbb{K}$, and $\left|a_{i}\right| \neq 1$ for $i=1,2, g: S \rightarrow X$, and

$$
\sup _{x \in S}\|g(x+2)-p g(x+1)+q g(x)\|<\varepsilon .
$$

Then there is a solution $f: S \rightarrow X$ of (4.1) that satisfies (1.8).

For instance, if $p=1$ and $q=-1$ (the case of the Fibonacci and Lucas numbers), we have the following.

Corollary 4.2. Let $p=1, q=-1, \varepsilon>0$, and $\left\{\alpha_{n}\right\}_{n \in \mathbb{N}_{0}}$ be a sequence in $X$ with

$$
\sup _{n \in \mathbb{N}_{0}}\left\|\alpha_{n+2}-p \alpha_{n+1}+q \alpha_{n}\right\| \leq \varepsilon .
$$


Then there is a sequence $\left\{\beta_{n}\right\}_{n \in \mathbb{N}_{0}}$ in $X$ such that

$$
\begin{aligned}
& \beta_{n+2}=p \beta_{n+1}-q \beta_{n}, \quad n \in \mathbb{N}_{0} \\
& \sup _{n \in \mathbb{N}_{0}}\left\|\alpha_{n}-\beta_{n}\right\| \leq(2+\sqrt{5}) \varepsilon .
\end{aligned}
$$

Proof. Note that $\left(\left|a_{1}\right|-1\right)\left(\left|a_{2}\right|-1\right)=2-\sqrt{5}$. Thus, by Proposition 4.1, there is a sequence $\left\{\beta_{n}\right\}_{n \in \mathbb{N}_{0}}$ in $X$ such that (4.4) is valid.

Remark 4.3. If $p=1$ and $q=-2$ (the case of Jacobsthal numbers), then one of the roots of (1.1) is equal to -1 and therefore (4.1) is not stable (see [28]), by which we mean that, for each $\varepsilon>0$, there is $g: S \rightarrow X$ such that $\sup _{x \in S}\|g(x+2)-p g(x+1)+q g(x)\|<\varepsilon$ and $\sup _{x \in S}\|g(x)-f(x)\|=\infty$ for every solution $f: S \rightarrow X$ of (1.6); for $S=\mathbb{N}_{0}$ such function $g$ can be chosen with, for example, $g(0)=0$ and $g(1)=1$ (in [28, the proofs of Lemma 2 and Theorem 4] take $\bar{y}_{0}:=1$ ).

\section{Acknowledgments}

This research was supported by the Basic Science Research Program through the National Research Foundation of Korea (NRF), funded by the Ministry of Education, Science, and Technology (no. 2010-0007143).

\section{References}

[1] D. H. Hyers, "On the stability of the linear functional equation," Proceedings of the National Academy of Sciences of the United States of America, vol. 27, pp. 222-224, 1941.

[2] R. P. Agarwal, B. Xu, and W. Zhang, "Stability of functional equations in single variable," Journal of Mathematical Analysis and Applications, vol. 288, no. 2, pp. 852-869, 2003.

[3] S. Czerwik, Functional Equations and Inequalities in Several Variables, World Scientific, River Edge, NJ, USA, 2002.

[4] D. H. Hyers, G. Isac, and Th. M. Rassias, Stability of Functional Equations in Several Variables, Progress in Nonlinear Differential Equations and Their Applications, 34, Birkhäuser, Boston, Mass, USA, 1998.

[5] S.-M. Jung, "Functional equation $f(x)=p f(x-1)-q f(x-2)$ and its Hyers-Ulam stability," Journal of Inequalities and Applications, vol. 2009, Article ID 181678, 10 pages, 2009.

[6] Z. Moszner, "On the stability of functional equations," Aequationes Mathematicae, vol. 77, no. 1-2, pp. 33-88, 2009.

[7] B. Paneah, "A new approach to the stability of linear functional operators," Aequationes Mathematicae, vol. 78, no. 1-2, pp. 45-61, 2009.

[8] L. Cădariu and V. Radu, "Fixed point methods for the generalized stability of functional equations in a single variable," Fixed Point Theory and Applications, vol. 2008, Article ID 749392, 15 pages, 2008.

[9] J. Chudziak, "Stability problem for the Gołąb-Schinzel type functional equations," Journal of Mathematical Analysis and Applications, vol. 339, no. 1, pp. 454-460, 2008.

[10] K. Ciepliński, "Stability of the multi-Jensen equation," Journal of Mathematical Analysis and Applications, vol. 363, no. 1, pp. 249-254, 2010.

[11] D. Miheț, "The probabilistic stability for a functional equation in a single variable," Acta Mathematica Hungarica, vol. 123, no. 3, pp. 249-256, 2009.

[12] J. Sikorska, "On a pexiderized conditional exponential functional equation," Acta Mathematica Hungarica, vol. 125, no. 3, pp. 287-299, 2009.

[13] S.-M. Jung, "Hyers-Ulam stability of Fibonacci functional equation," Bulletin of the Iranian Mathematical Society, vol. 35, no. 2, pp. 217-227, 282, 2009. 
[14] P. Ribenboim, My Numbers, My Friends, Popular Lectures on Number Theory, Springer, New York, NY, USA, 2000.

[15] K. Baron and W. Jarczyk, "Recent results on functional equations in a single variable, perspectives and open problems," Aequationes Mathematicae, vol. 61, no. 1-2, pp. 1-48, 2001.

[16] M. Kuczma, Functional Equations in a Single Variable, vol. 46 of Monografie Matematyczne, Polish Scientific Publishers, Warszawa, Poland, 1968.

[17] M. Kuczma, B. Choczewski, and R. Ger, Iterative Functional Equations, vol. 32 of Encyclopedia of Mathematics and Its Applications, Cambridge University Press, Cambridge, UK, 1990.

[18] S. Stević, "Bounded solutions of a class of difference equations in Banach spaces producing controlled chaos," Chaos, Solitons \& Fractals, vol. 35, no. 2, pp. 238-245, 2008.

[19] W. Hayes and K. R. Jackson, "A survey of shadowing methods for numerical solutions of ordinary differential equations," Applied Numerical Mathematics, vol. 53, no. 2-4, pp. 299-321, 2005.

[20] K. Palmer, Shadowing in Dynamical Systems, vol. 501 of Mathematics and Its Applications, Kluwer Academic Publishers, Dordrecht, The Netherlands, 2000.

[21] S. Y. Pilyugin, Shadowing in Dynamical Systems, vol. 1706 of Lecture Notes in Mathematics, Springer, Berlin, Germany, 1999.

[22] J. Brzdęk, D. Popa, and B. Xu, "Hyers-Ulam stability for linear equations of higher orders," Acta Mathematica Hungarica, vol. 120, no. 1-2, pp. 1-8, 2008.

[23] T. Trif, "Hyers-Ulam-Rassias stability of a linear functional equation with constant coefficients," Nonlinear Functional Analysis and Applications, vol. 11, no. 5, pp. 881-889, 2006.

[24] M. Fabian, P. Habala, P. Hájek, V. Montesinos Santalucía, J. Pelant, and V. Zizler, Functional Analysis and Infinite-Dimensional Geometry, CMS Books in Mathematics/Ouvrages de Mathématiques de la SMC, 8, Springer, New York, NY, USA, 2001.

[25] J. Ferrera and G. A. Muñoz, "A characterization of real Hilbert spaces using the Bochnak complexification norm," Archiv der Mathematik, vol. 80, no. 4, pp. 384-392, 2003.

[26] R. V. Kadison and J. R. Ringrose, Fundamentals of the Theory of Operator Algebras. Vol. I. Elementary Theory, vol. 100 of Pure and Applied Mathematics, Academic Press, New York, NY, USA, 1983.

[27] R. V. Kadison and J. R. Ringrose, Fundamentals of the Theory of Operator Algebras. Vol. I. Elementary Theory, vol. 15 of Graduate Studies in Mathematics, American Mathematical Society, Providence, RI, USA, 1997.

[28] J. Brzdȩk, D. Popa, and B. Xu, "Note on nonstability of the linear recurrence," Abhandlungen aus dem Mathematischen Seminar der Universität Hamburg, vol. 76, pp. 183-189, 2006.

[29] D. Popa, "Hyers-Ulam stability of the linear recurrence with constant coefficients," Advances in Difference Equations, vol. 2005, no. 2, pp. 101-107, 2005. 